grasse et, en même temps, limite, dans une large mesure, la tendance que peuvent avoir les grumeaux de caséine à s'agglomérer, de sorte que les échantillons, après plusieurs mois, sont non seulement intacts, mais peuvent facilement se réémulsionner, parfois par simple agitation, et ceci n'est pas un des moindres avantages.

Au total, l'association bichromate-alcool amylique réalise une protection totale de l'échantillon, chacun de ces deux produits étant insuffisants par lui-même et facilite énormément les opérations ultérieures.

L'alcool amylique est, par ailleurs, un produit non nocif, facile à manipuler, enfin sans action ni sur le lait lui-même ni sur la marche de l'analyse. Nous sommes convaincus que son emploi, surtout en période d'été ou dans les pays chauds, ne pourrait que donner des résultats satisfaisants.

Il serait pratique de l'utiliser soit, par exemple, sous forme d'ampoules de 1 à $2 \mathrm{~cm}^{3}$, soit avec un simple compte-gouttes.

\title{
LA FABRICATION D'ACIDE LACTIQUE PUR
}

par

\section{G. GENIN}

Ingénieur E. P. C.

Nous avons déjà à plusieurs reprises insisté sur l'intérêt que prend actuellement l'acide lactique en chimie industrielle et sur les procédés de fabrication de cet acide ou de ses sels. L'acide lactique est en effet aujourd'hui préparé industriellement par fermentation de divers hydrates de carbone, comme par exemple le sucre de maĩs, les mélasses ou le lactose contenu dans le sérum du lait. La solution aqueuse d'acide lactique que l'on obtient au cours de ces fermentations et des opérations qui les prolongent, contient certaines impuretés inorganiques ou organiques comme par exemple des sulfates de calcium et de sodium, des sucres non fermentés, des matières colorantes assez foncées et certaines substances azotées. Ces solutions d'acide lactique brut peuvent être employées dans certaines industries, comme par exemple pour la teinture des tissus ou le tannage du cuir, mais il est certaines autres applications qui exigent l'emploi d'un acide parfaitement pur.

Au cours de ces dernières années, en effet, des applications nouvelles importantes ont été trouvées pour l'acide lactique, à condition que le produit employé soit plus pur que l'acide généralement fabriqué jusqu'à présent. C'est ainsi, par exemple, qu'on a envisagé l'emploi de l'acide pur comme plastifiant et comme 
catalyseur dans la préparation des résines phénoliques destinées à être coulées. II faut dans ce cas que l'acide utilisé soit de bonne qualité et pratiquement exempt de sulfates et de chlorures de métaux lourds, ainsi que de produits qui peuvent communiquer à l'objet moulé une odeur ou un goût désagréable.

Aux Etats-Unis, où la fabrication de l'acide lactique s'effectue déjà sur une grande échelle, on trouve 4 qualités différentes d'acide lactique : l'acide brut qui peut être à 22 , à 44 ou à $50 \%$; l'acide comestible qui peut être à 44 et à $50 \%$; l'acide de qualité matière plastique dont la teneur est supérieure à $50 \%$ et l'acide de la pharmacopée américaine à $85 \%$. En ce qui concerne la qualité alimentaire de cet acide, aucune spécifieation particulière ne lui est appliquée, si ce n'est que les règlements généraux applicables aux aliments sont naturellement valables pour ce produit. Il ne doit donc contenir aucune substance susceptible d'être un danger pour la santé des consommateurs. C'est généralement l'usager de ce produit qui fixe lui-même les qualités auxquelles il doit répondre et ces qualités se rapportent surtout à la coloration, à l'odeur et à la saveur du produit.

En ce qui concerne l'acide lactique, destiné à la fabrication des résines phénoliques transparentes pouvant être coulées, les spécifications sont beaucoup plus sévères, et voici par exemple des standards de qualité applicables à cet acide. La teneur en acide doit être supérieure à $50 \%$ et la teneur en chlorure (calculée en chlore), inférieure à 5 parties par million, la teneur en sulfate (calculée en $\mathrm{SO}^{4}$ ), inférieure à 50 parties par million; enfin la teneur en cendres inférieure à $0,05 \%$.

Ce produit ne doit contenir que des traces de fer et il est tout à fait essentiel que cette condition soit réalisée, car ce métal ou ses composés ont la propriété de réagir avec le phénol pour. former des substances colorées. La présence d'autres sels métalliques est d'ailleurs tout aussi défavorable, ear ees produits sont insolubles dans la résine et entraînent l'apparition d'un louche ou d'une opalescence dans le produit fini.

On voit que l'acide lactique destiné à être utilisé dans l'industrie des matières plastiques doit répondre à des conditions excessivement sévères et on conçoit que ce produit soit d'une préparation difficile. L. T. Sмith et H. V. Claborn, du Bureau of Dairy Industry du Ministère des Etats-Unis [1] ont exposé récemment les divers procédés que l'on peut employer pour la préparation de l'acide lactique pur et nous emprunterons à ces auteurs les renseignements qui suivent :

Les procédés dont on dispose aujourd'huri pour la fabrieation de l'acide lactique pur sont les suivants : 


\section{Purification par cristallisation du lactate de calcium}

Le lactate de calcium brut qui précipite par concentration des solutions provenant de la fermentation des hydrates de carbone est dissous et soumis à des cristallisations successives afin de le purifier. Le produit suffisamment pur est alors dissous, puis acidifié selon la méthode indiquée par OLIve [2] ou par Burton [3]. On obtient dans ces conditions et suivant le degré de purification du lactate de, calcium, de l'acide lactique pouvant être utilisé pour l'alimentation ou répondant aux spécifications de la Pharmacopée britannique ou encore susceptible d'être employé dans la préparation des résines phénoliques colorées.

\section{Purification du lactate de zinc}

On peut également obtenir de l'acide lactique chimiquement pur en transformant le lactate de calcium en sel de zinc correspondant, en utilisant à cet effet du carbonate ou du sulfate de zinc. On purifie alors le lactate de zine par cristallisation et on le dissout dans l'eau. Le zinc est ensuite précipité par addition d'hydrogène sulfuré et la solution d'acide lactique préparée dans ces conditions est décolorée par le charbon animal, filtrée et le filtrat est évaporé dans le vide [4].

Le sel de zinc convient particulièrement bien à cette préparation, car il cristallise beaucoup mieux que n'importe quel autre lactate.

\section{Extraction par les solvants de l'acide lactique contenu dans les solutions aqueuses}

On a envisagé l'emploi de divers solvants pour extraire l'acide lactique contenu dans une solution aqueuse, en tenant compte du coefficient de distribution de cet acide, entre l'eau et le solvant. En particulier, JENEMANN [5] a imaginé un procédé continu d'extraction en contre-courant dans lequel on utilise l'éther isopropylique comme solvant. Mais ce procédé n'est pas efficace, il est coûteux et en outre il présente de grands dangers, car l'éther isopropylique est inflammable et risque en outre de former des peroxydes explosibles.

\section{Oxydation des impuretés organiques}

On peut réaliser une purification partielle de l'acide lactique en soumettant les solutions brutes de l'acide industriel ou encore de ses sels, à une légère oxydation [6]. Comme agent oxydant, on peut utiliser des solutions d'hypochlorite de calcium et de sodium, de chromate et de permanganate de potassium, d'eau oxygénée ou d'acide nitrique; on peut également utiliser le chlore ou l'ozone à l'état gazeux. On est parvenu de cette façon à préparer des qualités alimentaires d'acide lactique. 


\section{Distillation fractionnée}

Lorsqu'on chauffe de l'acide lactique et en particulier un produit contenant certaines de ses impuretés naturelles, il tend à se décomposer et il n'est done pas possible de purifier cet acide par une distillation effectuée sans précaution particulière. On a done cherché à améliorer les procédés habituels de distillation, en opérant par exemple dans le vide, ou en effectuant la distillation au moyen de vapeur surchauffée, d'air chaud ou de gaz inerte [7]. Aucune de ces techniques n'a trouvé d'application industrielle.

\section{Séparation et hydrolyse des esters lactiques}

Les lactates de méthyle, d'éthyle, de propyle ou d'isopropyle sont très facilement saponifiés par la vapeur ou l'eau chaude à la pression atmosphérique [8]. On recueille alors le résidu de eette saponification, on le concentre dans le vide et on obtient dans ces conditions un acide lactique chimiquement pur, parfaitement incolore qui répond à toutes les spécifications imposées aux qualités les plus pures de ce produit.

Les techniciens du Bureau of Dairy Industry ont étudié d'une façon approfondie la question de l'éthérification de l'acide lactique et de l'hydrolyse des esters ainsi préparés. On a constaté en particulier qu'en partant de lactate de calcium ou de lactate de sodium brut, en ajoutant un alcool et en chauffant en présence d'un excès d'acide sulfurique, on obtient avec un rendement de 70 à $90 \%$ par rapport au rendement théorique un ester lactique pur dont le prix de revient, étant donné la simplicité de ce produit, n'est pas prohibitif.

Voici d'ailleurs le mode opératoire conseillé par SмIтH et CLABORN pour procéder à cette opération.

On part d'une molécule de lactate de calcium ou de 2 molécules de lactate de sodium, de préférence exempt d'humidité, que l'on dissout dans 10 à 20 molécules d'alcool méthylique. Les substances insolubles sont séparées par filtration. Le filtrat est acidifié avec une quantité suffisante d'acide sulfurique pour libérer l'acide lactique, pour former du sulfate de calcium ou de sodium et pour eatalyser l'éthérification. La masse est alors chauffée au reflux pendant 4 à 8 heures, afin de procéder à cette éthérification, puis filtrée afin d'éliminer les substances précipitées.

On élimine alors l'excès d'alcool éthylique par distillation à la pression atmosphérique, puis on distille le résidu dans le vide afin de recueillir l'eau et la majeure partie de l'ester. En opérant de cette façon, les risques d'hydrolyse de l'ester sont réduits au minimum. La distillation doit d'ailleurs être arrêtée avant qu'il y ait dans la chaudière de distillation commencement de earbonisation 
due à l'excès d'acide sulfurique. Le résidu qui subsiste dans cette chaudière pourra être utilisé de deux façons différentes : ou bien on pourra l'additionner d'acide sulfurique et l'ajouter à une nouvelle fabrication, en le considérant comme matière première, ou bien il pourra être extrait au moyen d'alcool méthylique frais, cet alcool étant utilisé pour une nouvelle estérification.

Le distillat est alors dilué par 2 à 3 fois son volume d'eau distillée et on le sépare par distillation fractionnée, en opérant lentement à la pression atmosphérique et en utilisant une colonne en matériaux non corrosifs. Lorsque le lactate de méthyle a été hydrolysé et que l'alcool méthylique a été éliminé par distillation, la solution d'acide lactique qui reste dans la chaudière est évaporée dans le vide, jusqu'à ce que l'on obtienne une concentration voulue en acide lactique.

A la suite des travaux qui ont été entrepris au Bureau of Dairy Industry par les chimistes américains, on a pu établir que la préparation de l'acide lactique chimiquement pur par purification et hydrolyse des esters lactiques et plus particulièrement du lactate de méthyle, s'est révélée comme le procédé le plus efficace et le plus économique pour l'obtention industrielle de ce produit. Ces esters sont très facilement et très économiquement préparés en partant du lactate de sodium on du lactate de calcium, ces produits étant obtenus eux-mêmes dans des conditions économiques, en utilisant comme matière première le sérum du lait. Si on opère au cours de ces diverses opérations dans des conditions appropriées, on parvient à obtenir un rendement en acide lactique égal à $85 \%$ du rendement théorique. Toutes les opérations qui interviennent dans la préparation des divers produits intermédiaires sont des opérations classiques en chimie industrielle, consistant la plupart du temps en une simple distillation. L'outillage nécessaire, qu'il s'agisse d'une opération de laboratoire ou d'une fabrication industrielle, est simple et relativement peu coûteux et ce procédé ne fait intervenir aucun solvant inflammable ou explosible.

II est surtout conseillé pour la préparation du produit : le lactate de méthyle. En effet, l'alcool méthylique comme le lactate de méthyle sont des corps à poids moléculaire bas et en outre à point d'ébullition peu élevé. Il en résulte que les dépenses de combustible sont réduites au minimum pour effectuer la distillation et la séparation par fractionnement de ces produits. En outre, les droits fiscaux qui frappent l'alcool éthylique ne s'appliquent pas à l'alcool méthylique ou au lactate de méthyle. Enfin, le lactate de méthyle est facilement hydrolysé et sa récupération est aisée. 
No I - Ch. Porcher. Le lait au point de vue colloidal. Recherches sur le mécanisme de.l'action de la présure.

I vol., broch., in-80, 530 p., avec nombreux graphiques Epuisé ; a paru dans "Le Lait" I929 et I 930. - I7 nos.

Prix : 200 francs.

$\mathrm{N}_{2} 2$ - Ch. PORcher. Le procès de la matière grasse. Epuisé ; a paru dans " Le Lait" 1925 .

Prix : I 50 francs.

No 3 - Ch. Porcher. Le lait desséché. $2^{\mathrm{e}}$ éd., I vol. petit in $-4^{\circ}, x x-298$ p., 9 fig., 8 pl. hors texte.

Epuisé.

No 4 - A. Leroy. Ge que doit savoir un bon contrôleur' laitier. $2^{\mathrm{e}}$ éd., I 44 p., 16 graphiques, $7 \mathrm{pl}$. hors texte.

Prix : 36 francs

$\mathrm{N}^{0} 5$ - E. Muffet, Docteur-Vétérinaire. Contribution à l'étude de la rétention lactée. Le sort de la caséine dans ce processus.

Epuisé.

No 6 - F. PEyronny, Docteur-Vétérinaire. Les variations du taux butyreux des laits individuels, I026. Epuisé

No 7 - A. Lataix, Docteur-Vétérinaire, Contribution à l'étude de la réfractométrie des lacto-sérums, I928.

Prix : I5 francs.

No $8-\mathrm{R}$ Bus, Docteur-Vétérinaire. L'allaitement artificiel chez les animaux domestiques, 1928 .

Epuisé.

No 9 - R. Desfougères, Docteur-Vétérinaire. La machine à traire, I930.

Epuisé.

No ro - R. Lотн, Docteur-Vétérinaire. Les malpropretés visibles du lait, I930. Deux pl. en noir et I6 pl, en couleurs.

Epuisé.

No II - G. Belle, Docteur-Vétérinaire. Recherches sur la croissance de quelques mammifères, I930. Prix : 27 francs

No I2 - F. Sansonetri, Docteur en pharmacie. Sur les flores bactérienne et fongique des caillés de lait, I930.

Prix : 27 francs.

No I3 - M BEAU, Ingénieur agronome. La caséine. 2 parties ne pouvant être vendues séparément. Réduction io \% aux abonnés de la revue $L e$ Lait. Prix : 60 francs.

No I4 - J. BRIgando. Docteur ès sciences, Université de Lyon. Recherches sur la caséine, I933.

a paru dans Le Lait, no8 I26, I27, I28, I29, I30, I933.

Epuisé

Prix : 90 francs.

No I5 - J. Pien, R. MARtin et M. Bergier. Examen chi mique de la qualité des caséines lactiques. I vol. : 27 francs.

Pour toutes ces publications, les prix ci-dessus s'entendent port en sus 


\section{RÉFÉRENCES}

[1] L. T. Smith et H. V. Claborn. Ind. Eng. News Ed., t. XVII, p. 641, 1939.

[2] T. R. OLIve. Chem. and Met. Eng., t. XLIII, p. 480, 1936.

[3] L. V. Burton. Food Ind, t. IX, p. 634, 1937.

[4] Standard Brands Inc. Brevet américain 2.024.565 du 17 décembre 1936.

[5] Jenemann J. A. et Grasselli Chemical Company. Brevet américain 1.906 .068 du 25 avril 1933.

[6] E. I. DU Pont de Nemours and Co. Brevet américain 2.013.096 du 3 septembre 1935 .

Id. Brevet américain 2,101.998 du 14 décembre 1938 .

Grasselli Chemical Company. Brevet américain 2.013.082 du 3 sep. tembre 1935 .

[7] E. I. DU Pont de Nemours AND Co. Brevet américain 2.013.896 et 2.013.104 du 3 septembre 1935 .

[8] Chemische Fabrik Güstrow. Brevet allemand 171.835 du 25 juin 1906. Chemischewerke Vorm. Dr Heinrich VYK. Brevet allemand 278.487 du 26 septembre 1914.

\section{REVUE}

\section{L'INDUSTRIE LAITIËRE A L'ÉTRANGER}

par G. GÉNIN

Ingénieur Chimiste E. P. C.

ANGLETERRE

\section{L'alimentation du bétail en temps de guerre}

Le Ministère de l'Agriculture a rappelé aux fermiers anglais qu'avant guerre, l'Angleterre importait de 7 à 8 millions de tonnes de produits d'alimentation pour le bétail, les vaches laitières eonsommant environ le quart de ces importations. Il est impossible maintenant de poursuivre cette politique et les importations doivent être obligatoirement réduites, alors qu'au même moment, on demande aux fermiers d'augmenter leur production de lait.

Ce double résultat ne peut être obtenu que par l'amélioration des pâturages qui constituent encore aujourd'hui la forme la plus économique et la plus efficace d'aliment facilitant la production du lait et dans la culture sur le sol anglais de produits susceptibles de convenir à l'alimentation du bétail,

Désormais, les concentrés ne seront délivrés que pendant l'hiver et, même à cette époque de l'année, il faudra remplacer le maïs et les tourteaux importés par des produits anglais, comme par exemple la pulpe de betterave séchée, les sous-produits de l'industrie de la brasserie, etc.

\section{Un nouvel homogénéiseur}

La question de l'homogénéisation dans l'industrie laitière prend une importance de plus en plus grande et non seulement, pour 